\section{INSULIN RESISTANCE IMPAIRS ANGIOGENIC PROGENITOR CELL FUNCTION AND DELAYS ENDOTHELIAL REPAIR FOLLOWING VASCULAR INJURY}

doi:10.1136/heartjnl-2011-300110.3

M B Kahn, N Yuldasheva, R Cubbon, J Surr, S Rashid, H Viswambharan, H Imrie, A Abbas, A Rajwani, M Gage, M T Kearney, S Wheatcroft. Leeds University, Leeds, UK

Introduction Insulin-resistance, the primary metabolic abnormality underpinning type-2-diabetes mellitus (T2DM) and obesity, is an important risk factor for the development of atherosclerotic cardiovascular disease. Circulating-angiogenic-progenitor-cells (APCs) participate in endothelial-repair following arterial injury. Type-2 diabetes is associated with fewer circulating APCs, APC dysfunction and impaired endothelial-repair. We set out to determine whether insulin-resistance per se adversely affects APCs and endothelialregeneration.

Research Design and Methods We quantified APCs and assessed APC-mobilisation and function in mice hemizygous for knockout of the insulin receptor (IRKO) and wild-type (WT) littermate controls. Endothelial-regeneration following femoral artery wire-injury was also quantified at time intervals after denudation and following APC transfusion.

Results The metabolic phenotype of IRKO mice was consistent with compensated insulin resistance, with hyperinsulinaemia after a glucose challenge but a normal blood glucose response to a glucose tolerance test. IRKO mice had fewer circulating Sca-1+/Flk-1+ APCs than WT mice at baseline. Culture of mononuclear-cells demonstrated that IRKO mice had fewer APCs in peripheral-blood, but not in bone-marrow or spleen, suggestive of a mobilisation defect. Defective VEGF-stimulated APC mobilisation was confirmed in IRKO mice, consistent with reduced eNOS expression in bone marrow and impaired vascular eNOS activity. Paracrine-angiogenicactivity of APCs from IRKO mice was impaired compared to those from WT animals. Endothelial-regeneration of the femoral artery following denuding wire-injury was delayed in IRKO mice compared to WT (re-endothelialised area $35.8 \pm 4.8 \%$ vs $66.6 \pm 5.2 \%$ at day 5 following injury and $35.6 \pm 4.8 \%$ vs $59.8 \pm 6.6 \%$ at day 7 ; $\mathrm{P}<0.05)$ (Abstract C Figure 1A). Transfusion of mononuclear-cells from WT mice normalised the impaired endothelial-regeneration in IRKO mice $(57 \pm 4 \%$ vs $25 \pm 5 \%$; $<<0.002)$. Transfusion of c-kit+ bone-marrow cells from WT mice also restored endothelial-regeneration in IRKO mice $(62 \pm 2 \%$ vs $25 \pm 5 \%$; $<0.002)$. However, transfusion of c-kit + cells from IRKO mice was less effective at improving endothelial-repair ( $62 \pm 2 \%$ vs $45 \pm 4 \%$; $<<0.02$ ) (Abstract C Figure 1B).

Conclusions Insulin-resistance impairs APC function and delays endothelial-regeneration following arterial injury. These findings support the hypothesis that insulin-resistance per se is sufficient to jeopardise endogenous vascular repair. Defective endothelial-repair
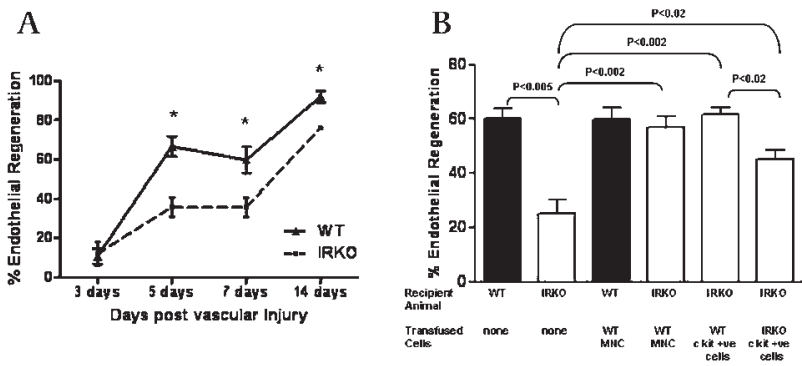

Abstract C Figure 1 (A) Time-dependent endothelial regeneration following vascular injury ( $n=5$ mice per group; ${ }^{*}$ denotes $\left.p<0.05\right)$. (B) Effects on endothelial regeneration 5 days after wire-injury of transfusion of spleenderived MNCs or BM-derived c-kit (CD117) + ve cells from WT or IRKO mice ( $n=4$ mice per group). may be normalised by transfusion of APCs from insulin-sensitive animals but not from insulin-resistant animals. These data may have important implications for the development of therapeutic strategies for insulin-resistance associated cardiovascular disease.

\begin{tabular}{l}
\hline UPTAKE OF ULTRASMALL SUPERPARAMAGNETIC \\
PARTICLES OF IRON OXIDE PREDICTS GROWTH IN \\
ABDOMINAL AORTIC ANEURYSMS: A PILOT STUDY
\end{tabular}

doi:10.1136/heartjnl-2011-300110.4

J M J Richards, S I Semple, T J Mac Gillivray, C Gray, J P Langrish, M Williams, M Dweck, W Wallace, G McKillop, R T A Chalmers, 0 J Garden, D E Newby. University of Edinburgh, Edinburgh, UK

Background Prediction of abdominal aortic aneurysm (AAA) expansion and rupture is challenging and currently relies on serial measurements of maximum aneurysm diameter. Using ultrasmall superparamagnetic particles of iron oxide (USPIO) and MRI, we aimed to assess whether areas of cellular inflammation correlated with the rate of abdominal aortic aneurysm expansion.

Methods and Results An image acquisition and data analysis algorithm for the detection of focal USPIO accumulation in tissues was developed. Patients ( $n=29 ; 27$ male; aged $70 \pm 5$ years) with asymptomatic AAA $(4.0-6.6 \mathrm{~cm})$ were recruited from an outpatient surveillance programme and underwent 3T MRI before and 24-36 h after administration of USPIO. The change in $\mathrm{T} 2 *$ value on $\mathrm{T}^{*}$ weighted imaging was used to detect accumulation of USPIO within the abdominal aortic aneurysm. Histology of aortic wall tissue samples confirmed co-localisation and uptake of USPIO in areas with macrophage infiltration. Patients were classified into one of three groups on the basis of imaging findings (Abstract D Figure 1). Group 1: periluminal USPIO uptake only. Group 2: USPIO uptake throughout the thrombus. Group 3: USPIO uptake in the aortic wall. Patients in group 3 with distinct mural uptake of USPIO had a threefold higher growth rate $(\mathrm{n}=13 ; 0.66 \mathrm{~cm} / \mathrm{yr} ; \mathrm{p}=0.020)$ than those with no (Group $1 ; \mathrm{n}=7$; $0.22 \mathrm{~cm} / \mathrm{yr}$ ) or non-specific USPIO uptake (Group $2 ; \mathrm{n}=9 ; 0.24 \mathrm{~cm} / \mathrm{yr}$ ) despite having similar aneurysm diameters $(5.4 \pm 0.6,5.1 \pm 0.5$ and $5.0 \pm 0.5 \mathrm{~cm}$ respectively; $\mathrm{p}>0.05)$ and patient characteristics $(p>0.05)$. In one patient with an inflammatory aneurysm, USPIO uptake and inflammation extended beyond the aortic wall into surrounding tissues.

Conclusion USPIO uptake in the aortic wall detects cellular inflammation in patients with AAA and appears to predict more rapidly progressive AAA expansion. This technique therefore holds major promise as a new method of risk-stratifying patients with AAA that extends beyond the simple anatomical measure of aneurysm diameter
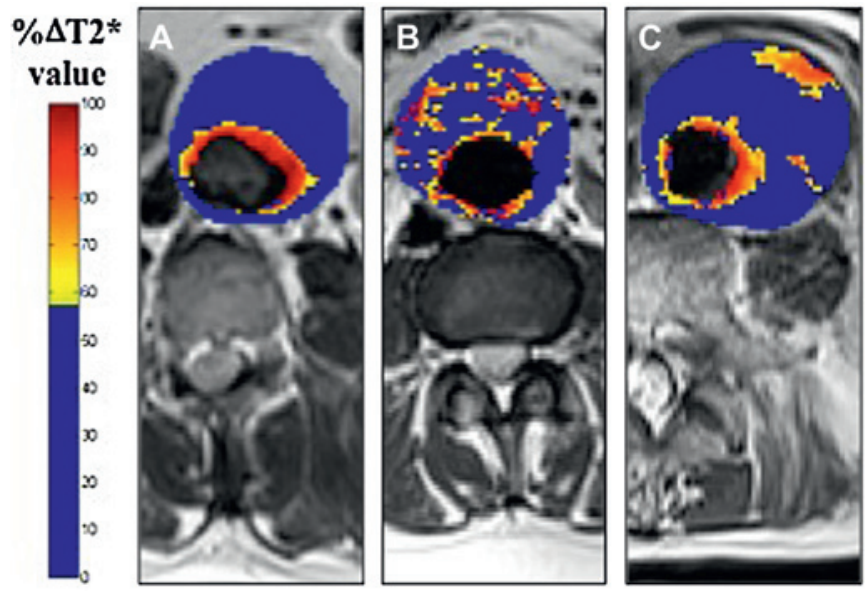

Abstract D Figure 1 


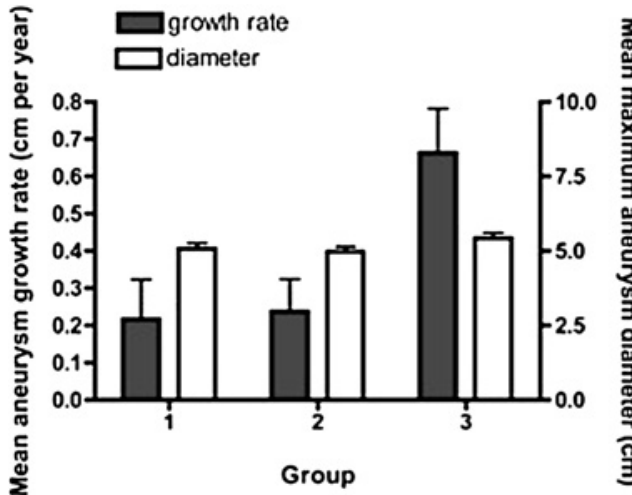

Abstract D Figure 2

\section{INTEGRATIVE GENOMICS APPROACHES IDENTIFY NEW GENES CONTROLLING HEART RATE}

doi:10.1136/heartjnl-2011-300110.5

${ }^{1,2} \mathrm{~J} S$ Ware, ${ }^{3} \mathrm{H}$ Dobrzynski, ${ }^{4} \mathrm{M}$ Pravanec, ${ }^{1} \mathrm{P} \mathrm{J}$ Muckett, ${ }^{1} \mathrm{~S}$ Wilkinson, ${ }^{5} \mathrm{Y}$ Jamshidi, ${ }^{1} \mathrm{~T} J$ Aitma, ${ }^{6} \mathrm{~N}$ S Peters, ${ }^{1,2} \mathrm{~S}$ A Cook. ${ }^{1} \mathrm{MRC}$ Clinical Sciences Centre, Imperial College London, London, UK; ${ }^{2}$ National Heart \& Lung Institute, Imperial College London, London, UK; ${ }^{3}$ School of Medicine, University of Manchester, Manchester, UK; ${ }^{4}$ Institute of Physiology, Czech Academy of Science, Prague, UK; ${ }^{5}$ Division of Clinical Developmental Sciences, St. George's University of London, London, UK; ${ }^{6}$ National Heart \& Lung Institute, Imperial College London, London, UK

Introduction Heart rate (HR) is a fundamental measure of cardiac function, and is of prognostic and therapeutic significance. We applied genetic and genomic approaches to identify new loci and genes controlling HR in a rat model that has previously been used to find human cardiovascular disease genes.

Methods Telemetric aortic pressure transducers were implanted into 226 animals from 33 rat strains: the Brown Norway, the Spontaneously Hypertensive Rat, and 31 strains from a recombinant inbred panel derived from these parental strains and HR was measured over several weeks. Statistical analyses were carried out using the $\mathrm{R}$ package, and quantitative trait loci (OTL) identified by linkage mapping using OTL Reaper. Potential covariates of HR were analysed in SPSS. The sinus node (SN) and right atria (RA) of 20 rats were microdissected (Abstract E Figure 1). Gene expression data were generated with the Affymetrix Rat Gene 1.0 ST microarray and analysed using Bioconductor. Differentially expressed genes were identified using SAM \& Limma. Genes in the QTL that were expressed in the $\mathrm{SN}$ were resequenced to identify potential causative sequence variants.

Results Narrow sense heritability of HR in this population was $51 \%$, suggesting a large genetic contribution to HR. Linkage mapping identified a region on rat chromosome 13 controlling HR, with peak LOD score 6.7 (Abstract E Figure 2A). This QTL has not previously been identified in human, rat or mouse. Mean nocturnal HR in strains carrying the SHR allele was 388, compared with 357 in BN-like strains; an allelic effect of $31 \mathrm{bpm}(8.7 \%, \mathrm{p}<0.00005)$ that is equivalent to $5-9 \mathrm{bpm}$ in humans, corresponding to a decreased risk of cardiovascular death of $10 \%-29 \%$. Two independent approaches (linear regression modelling \& correlation analysis) confirmed that this effect was independent of potential physiological covariates, suggesting that the effect may be intrinsic to the heart, rather than due to neurohumeral influences. We have generated the first genomewide transcript expression profile of the $\mathrm{SN}$. Three genes at the new HR locus were enriched in the SN
(Abstract E Figure 2B) and potentially causative sequence variants in these 3 candidate genes have been identified. We have translated these findings to humans using data from a genome-wide association study population.

Conclusions We have identified a new genomic locus for HR, which does not contain genes in pathways already known to determine HR. We prioritised three candidate genes at the locus, which may be targets for therapeutic modulation of HR in patients with heart disease.

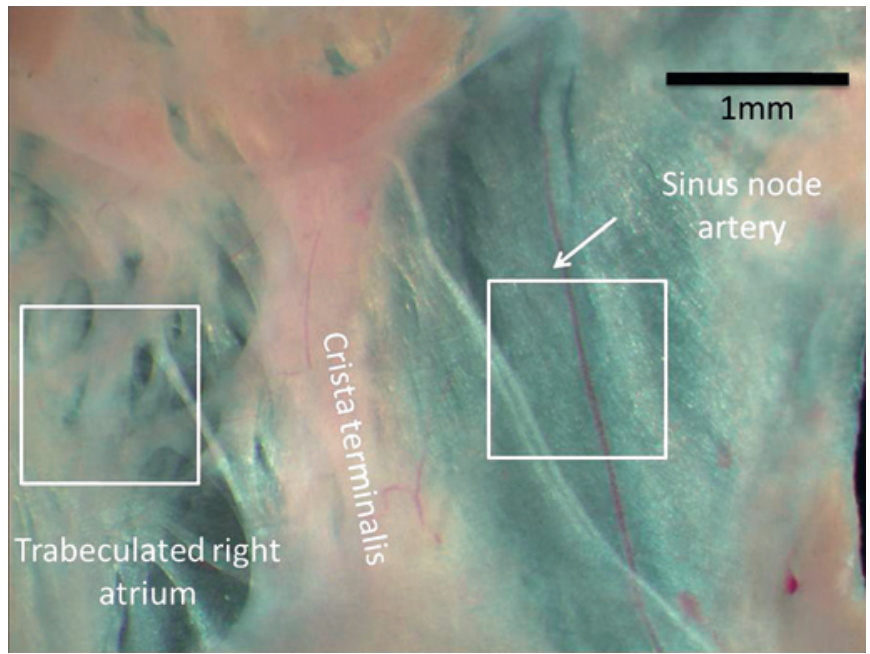

Abstract E Figure 1 Small $\left(1 \mathrm{~mm}^{2}\right)$ pieces of tissue were isolated from the rat SN and distant trabeculated RA and RNA extracted for gene expression profiling.

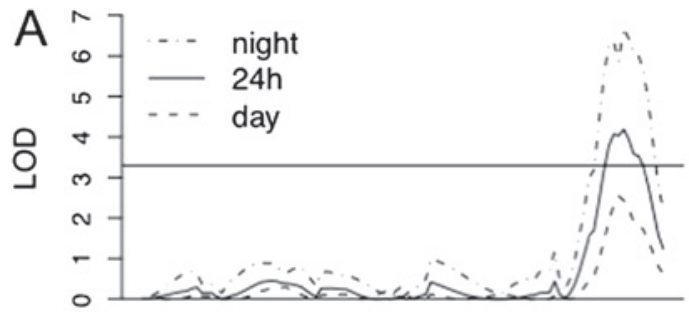

Chromosome 13

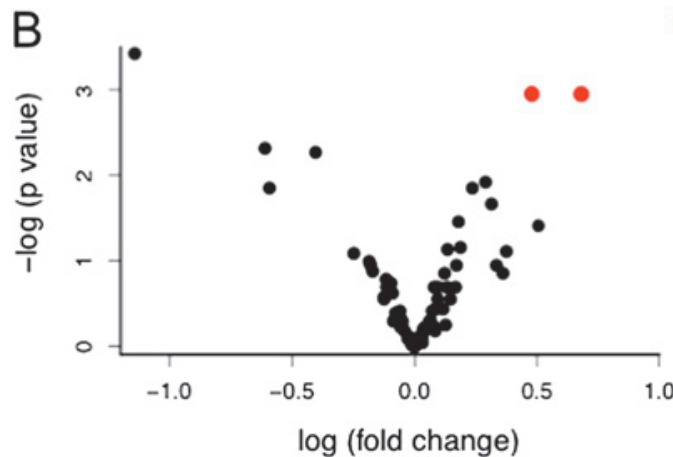

Abstract E Figure 2 (A) Interval mapping revealed a linkage peak on chr13. Linkage was strongest for nocturnal HR, with a LOD score of 6.7 ( $p>0.00005)$. The horizontal line approximates to genomewide significance. (B) A volcano plot showing 3 genes significantly enriched in SN compared with RA. 